\title{
Anaheim Pepper (Capsicum annuum L.) Production on Substrates under Greenhouse Conditions
}

\author{
Diego Nafarrate-Ramos', Bardo Heleodoro Sánchez-Soto ${ }^{1}$, Fidel Núñez-Ramirez², \\ Gabriel Antonio Lugo-García1, Víctor Ramón Sarabia-Perea1, Salomón Buelna-Tarín1 \\ ${ }^{1}$ Facultad de Agricultura del Valle del Fuerte, Universidad Autónoma de Sinaloa, Juan José Ríos, México \\ ${ }^{2}$ Instituto de Ciencias Agrícolas, Universidad Autónoma de Baja California, Mexicali, México \\ Email: diego.nafarrate@gmail.com
}

Received 9 May 2016; accepted 13 June 2016; published 17 June 2016

Copyright (C) 2016 by authors and OALib.

This work is licensed under the Creative Commons Attribution International License (CC BY).

http://creativecommons.org/licenses/by/4.0/

(c) (i) Open Access

\section{Abstract}

The use of substrates in the vegetable production has benefits because they are involved in the availability of moisture and nutrient and they have a better handling. By this in the present study has been evaluated the production of Anaheim pepper variety Azteca F1, farmed in regional substrates like sand (Substrate A), sandy clay soil (Substrate B), and a composite substrate (Substrate C: $40 \%$ sand, $40 \%$ sandy clay, $20 \%$ compost). The experimental design was randomized blocks with 10 repetitions. The variables were submitted to analysis of variance, Tukey test and correlations with statistical package SAS version 9.0. In general, the plants farmed in the substrate $C$ are those with the highest values in plant height, number of flowers, fruits, length of fruits and yield. Therefore, the use of substrates particularly the substrate $C$ helps the growth and development of Anaheim pepper plants, it even increases the yield because it has a better control over the unlike soil.

\section{Keywords}

Vegetal Nutrition, Protected Agriculture, Soilless

Subject Areas: Agricultural Science

\section{Introduction}

El incremento de la población mundial obligaal sector agrícola a generar nuevas tecnologías con la finalidad de aumentar el rendimiento hortícola por unidad de superficie y la calidad de productos alimenticios para el mercado demandante [1]. El chile es uno de los cultivos agrícolas más importantes en México y el mundo. Sus fru- 
tos se consumen tanto en fresco comoen seco con el fin de proporcionar color, sabor y aroma a infinidad de platillos, lo anterior lo sitúa entre las principales especias. México es el país con la mayor diversidad de Capsicum annuum L., y su cultivo se realiza prácticamente en todo el territoriocon diferentes sistemas de producción y problemas diversos. Por ello, es de suma importancia la generación de información sobre el cultivo del chile para cada condición en particular [2].

El sistema de producción mediante el uso de invernaderos permite obtener mayor rendimiento y mejor calidad de frutos, asimismo, se consigue obtenerfruta en temporadas cuando no es posible a campo abierto, lo que se traduce en mejores precios en el mercado. Adicionalmente tiene el atributo de lograr un ahorro de agua superior al $30 \%$ [3].

Una de las ventajas que representa el uso de los sustratos regionales es la disponibilidad y un menor costo, y más aún los de origen orgánico dada la tendencia al manejo de sistemas de producción con enfoque sustentable [4].

Para el desarrollo y crecimiento de plántulas, el sustrato empleado es un factor fundamental puesto que éste contribuye en la calidad de lasmismas. Hartmann y Kester (2002) [5], mencionan que actualmente existe una gran cantidad de materiales que pueden ser utilizados para la elaboración de sustratos y su elección dependerá de la especie vegetal a propagar, tipo de propágulo, época de siembra, sistema de propagación, costo, disponibilidad y características propias del sustrato. Sin embargo, desde el punto de vista medioambiental los criterios más importantes para la elección de un material como sustrato en cultivos sin suelo son: su durabilidad y capacidad para ser reciclado posteriormente [6].

Los sustratos comerciales como peat moss, lana de roca, entre otros, son de alto costo, por lo que su uso, a pesar de sus grandes ventajas, representa un aumento en el valor de producciónpara aquellos agricultores con poca capacidad adquisitiva que desean cambiar a este esquema de produccióny se convierte en impedimento. Por su parte, en la región Norte de Sinaloa tanto la arena, el suelo arcillo-arenoso como la composta son abundantes y de fácil adquisición debido a que son muy económicos, cualidades que pueden representar una opción viable para este tipo de productores.

Por lo antes mencionado, el objetivo de la investigación fue evaluar la producción de chile Anaheim var. Azteca cultivado en tres sustratos bajo condiciones de invernadero.

\section{Materiales Y Métodos}

La presente investigación se realizó durante el ciclo otoño-invierno del ciclo 2014-2015en un invernadero tipo "Batisierra" con techo cubierto de polietileno y paredes de malla antiáfidos de 450 micras, instalado en la Facultad de Agricultura del Valle del Fuerte (FAVF) de la Universidad Autónoma de Sinaloa (UAS) en Juan José Ríos, Sinaloa, México (10849'23"O y 2545'57"N).

\subsection{Material Vegetal}

Se utilizó chile Anaheim variedad "Azteca F1".

Los sustratos usados fueron: arena, suelo arcillo arenoso y mezcla representados de la siguiente manera: 1) Sustrato A (Arena), 2) Sustrato B (49.21\% arena, $11.07 \%$ limo y $39.72 \%$ arcilla) y 3) Sustrato C (40\% Arena, $40 \%$ Arcillo arenoso, 20\% Composta). Las características de cada uno de ellos se presentan en el Cuadro 1.

\subsection{Sistema De Riego Y Fertirrigación}

Se utilizósistema de riego localizado, mismopor el cual se inyectó el fertilizante hacia cada una de las unidades experimentales. La fertilización utilizada durante el desarrollo del cultivo se encuentra representada en el Cuadro 2.

\subsection{Producción De Plántulas}

El día cinco de septiembre se sembró la semilla de chile Anaheim en los almácigosde producción de plántula para luego ser trasplantada a las bolsas con sustrato el día 17 de octubre.

\subsection{Diseño Experimental}

La evaluación de los sustratos se realizó utilizando tres bloques al azar ytres tratamientos, mientras que la un- 
Cuadro 1. Características fisicoquímicas de los sustratos.

\begin{tabular}{|c|c|c|c|}
\hline & Características & Valor & Interpretación \\
\hline \multirow{7}{*}{ ARENA } & $\mathrm{pH}$ & 7.1 & Ligeramente alcalino \\
\hline & Conductividad Eléctrica (dS/m) & 0.97 & Ligeramente salino \\
\hline & $\mathrm{CaCO}_{3}(\%)$ & 0.92 & Bajo \\
\hline & Densidad Aparente $\left(\mathrm{g} / \mathrm{cm}^{3}\right)$ & 1.70 & Alta \\
\hline & Arena (\%) & 49.21 & \\
\hline & Limo (\%) & 11.07 & Arcillo arenoso \\
\hline & Arcilla (\%) & 39.72 & \\
\hline \multirow{9}{*}{$\begin{array}{c}\text { SUELO } \\
\text { ARCILLO ARENOSO }\end{array}$} & $\mathrm{pH}$ & 6.64 & Ligeramente ácido \\
\hline & Materia Orgánica (\%) & 0.88 & Bajo \\
\hline & $\mathrm{CaCo}_{3}(\%)$ & 1.112 & Muy Bajo \\
\hline & Densidad Aparente $\left(\mathrm{gr} / \mathrm{cm}^{3}\right)$ & 1.42 & Alta \\
\hline & Conductividad Eléctrica (dS/m) & 1.424 & Ligeramente Salino \\
\hline & Materia Orgánica (\%) & $65-70$ & Alto \\
\hline & Nitrógeno $\left(\mathrm{N}_{2}\right)(\%)$ & $1.5-2$ & Medio \\
\hline & Fosforo $\left(\mathrm{P}_{2} \mathrm{O}_{5}\right)(\%)$ & $2-2.5$ & Alto \\
\hline & Potasio $\left(\mathrm{K}_{2} \mathrm{O}\right)(\%)$ & $1-1.5$ & Medio \\
\hline \multirow[t]{5}{*}{ COMPOSTA } & Relación C/N & $10-11$ & Buena \\
\hline & Ácidos Húmicos (\%) & $2.5-3$ & Alto \\
\hline & $\mathrm{pH}$ & 6.9 & Ligeramente ácido \\
\hline & Carbono Orgánico (\%) & $14-30$ & Alto \\
\hline & Calcio (\%) & $2-8$ & Alto \\
\hline
\end{tabular}

Cuadro 2. Fertilizantes utilizados durante el desarrollo del cultivo.

\begin{tabular}{cccc}
\hline Fecha & Fertilizante & Dosis & Veces por semana \\
9 Oct-29 Oct & $12-43-12$ & $1 \mathrm{~g} \cdot \mathrm{L}^{-1}$ & 3 \\
31 Oct-7 Nov & $12-43-12$ & $1.5 \mathrm{~g} \cdot \mathrm{L}^{-1}$ & 3 \\
10 Nov-4 Feb & $19-19-19$ & $1.5 \mathrm{~g} \cdot \mathrm{L}^{-1}$ & 3 \\
9 Oct-28 Nov & Nitrato de Calcio & $0.5 \mathrm{~g} \cdot \mathrm{L}^{-1}$ & 2 \\
9 Oct-28 Nov & Sulfato de Magnesio & $0.3 \mathrm{~g} \cdot \mathrm{L}^{-1}$ & 2 \\
2 Dic-4 Feb & Nitrato de Calcio & $0.6 \mathrm{~g} \cdot \mathrm{L}^{-1}$ & 2 \\
2 Dic-4 Feb & Sulfato de Magnesio & $0.8 \mathrm{~g} \cdot \mathrm{L}^{-1}$ & 1 \\
31 Dic-29 Ene & MKP & $0.6 \mathrm{~g} \cdot \mathrm{L}^{-1}$ & 1 \\
7 Ene-19 Ene & NKS & $1.2 \mathrm{~g} \cdot \mathrm{L}^{-1}$ & 2 \\
\hline
\end{tabular}

idad experimental consistió de 10 plantas separadas unas de otras $25 \mathrm{~cm}$ y $1.6 \mathrm{~m}$ entre camas para tener una plantación en bolsa a doble hilera. La población total de plantas fue de 50,000 plantas·ha ${ }^{-1}$. 


\subsection{Variables De Respuesta}

Las variables evaluadas fueron:altura de planta, diámetro tanto de tallocomo de frutos, número de hojas, botones florales, flores, y frutos.Así mismo se determinó el rendimiento y las variables antes mencionadas.A los 20 días después de trasplante se efectuaron los registros de número de hoja, altura de la planta, grosor de tallo; a los 32 días el número de botones florales y flores. Durante el periodo de producción se realizaron cuatro cortes a los 52, 70, 86 y 103 días después de trasplante.

\subsection{Análisis Estadístico}

Previo al análisis de varianza todos los datos se sometieron a la prueba de normalidad de Shapiro-Wilk. Con excepción del peso de frutos en el tercer corte que se realizaron con estadística no paramétrica mediante el ANDEVA Kruskal-Wallis debido a que los datos no erannormales. La comparación de medias se realizó con Tukey al 5\% de significancia. Las variables se analizaron con el programa SAS Institute Inc.

\section{Resultados}

El periodo de producción de chile Anaheim fue de 125 días después de trasplante, del 17 de octubre al 19 de febrero de 2015.

\subsection{Número De Hojas}

La arena presentó el mayor número de hojas que fue de 18.0 por planta, mientras que en suelo arcillo arenoso se obtuvieron 14.6. Sin embargo, no existe diferencia significativa en número de hojas $(P>0.05)$ entre tratamientos (Cuadro 3).

\subsection{Altura}

La mayor altura de planta fue de $15.23 \mathrm{~cm}$ y se logró en el sustrato C, en cambio los sustratos A y B mostraron $14.0 \mathrm{~cm}$ cada uno (Cuadro 3).

\subsection{Grosor De Tallo}

Los tallos de mayor grosor fueron de $3.30 \mathrm{~mm}$ y corresponde al sustrato $\mathrm{B}$, mientras que los de menor grosor de $2.94 \mathrm{~mm}$ se obtuvieronen el sustrato $C$, sin encontrar diferencia significativa $(P>0.05)$ entre los tratamientos (Cuadro 3).

\subsection{Botones Florales}

El mayor número de botones florales por planta se registró en el sustrato B con 57.80, por otra parte,la menor cantidad se presentó en el sustrato A con 17.73 (Cuadro 3).

\subsection{Número De Flores}

El sustrato C mostró la mayor cantidad de flores por plantacon un promedio de 2.53, mientras que el sustrato A presentó 0.53 (Cuadro 3).

Cuadro 3. Comparación de medias de las variables número de hojas, altura, diámetro de tallo y número de botones florales en chile jalapeño cultivado en tres sustratos.

\begin{tabular}{cccccc}
\hline Tratamiento & Número de hojas & Altura $(\mathbf{c m})$ & Diámetro del tallo (mm) & Botones florales & Flores \\
\hline Sustrato A & 18.00 & $14.00 \mathrm{~b}^{\beta}$ & 3.07 & $17.73 \mathrm{~b}$ & $0.53 \mathrm{~b}$ \\
Sustrato B & 14.66 & $14.06 \mathrm{~b}$ & 3.30 & $57.80 \mathrm{a}$ & $1.26 \mathrm{~b}$ \\
Sustrato C & 17.53 & $15.23 \mathrm{a}$ & 2.94 & $34.13 \mathrm{~b}$ & $2.53 \mathrm{a}$ \\
Probabilidad & 0.16 & 0.04 & 0.30 & $<0.01$ & 0.01 \\
\hline
\end{tabular}

${ }^{\beta}$ Letras distintas dentro de columnas indican diferencia significativa. 


\section{Corte 1}

El mayor número de frutos se encontró en las plantas del sustrato C con 1.8 frutos por planta, por su parte, la menor cantidad se produjo en el sustrato A (Cuadro 4 and Cuadro 5).

\section{Corte 2}

El mayor número de frutos por planta se registró en los sustratos B y C con 2.80, siendo el sustrato A el que menos frutos presento con 1 (Cuadro 4 and Cuadro 5).

\section{Corte 3}

No se obtuvieron diferencias significativas en ninguna de las variables evaluadas.

\section{Corte 4}

Los frutos más largos obtenidos en el experimento fueron de $14.96 \mathrm{~cm}$ en el sustrato C, mientras que el sustrato A presentó los frutos de menor longitud con $13.62 \mathrm{~cm}$ (Cuadro 4 and Cuadro 5).

\section{Discusión}

Los resultados indican que los sustratos tienen efecto sobre elcrecimiento y desarrollo de chile Anaheim cultivado bajo condiciones de invernadero, influyentanto en la altura de plantas, número de botones, número y largo de frutos, como en el rendimiento. Resultados que difieren a los de López et al (2013) [7], quienes no obtuvieron diferencia significativa del cultivar chile Anaheim en distintos sistemas de producción.

Se registródiferencia significativa para el primer y segundo corte, pero el tercer y cuarto corteno difieren entre sí. Los resultados coinciden con Klar y Jadoski (2004) [8], quienes en chile dulce bajo condiciones de estrés hídrico encontraron una reducción en el número de frutos; al igual que en trabajos realizados por Hassan et al. (2005) [9] y Gencoğlan et al. (2006) [10], al evaluar diferentes tratamientos de rendimiento en chile.

En el cuarto corte los resultados en el largo de frutos difieren significativamente conun promedio de $14.96 \mathrm{~cm}$, siendo el tercero, segundo y el primero similares uno del otro. La longitud promedio obtenida en este trabajo fue superior a la reportada por Ertek et al. (2007) [11], quienes obtuvieron un promedio de $12.8 \mathrm{~cm}$ de longitud en chile verde en condiciones de campo; estos resultados no coinciden con Wierenga (1983) [12], Demirtaş y Ayas (2009) [13], pues encontraron una disminución en la longitud de fruto a menor volumen de agua aplicada, como fue el caso del sistema arena. Se observaron diferencias entre los rendimientos de los tratamientos, de los cuales el sustrato C (Mezcla) registróla mayor cantidadcon $3.255 \mathrm{kgm}^{2}$, seguido de $2.354 \mathrm{Kgm}^{2}$ en el sustrato B y $1.929 \mathrm{Kg} \mathrm{m}^{2}$ en el sustrato A. Los resultados obtenidos indican ser superiores a los reportados por Flores (1996) [14], Ibarra et al.(2004) [15] y Santoyo et al. (2006) [16], los cuales obtuvieron un menor rendimiento bajo condiciones de campo en chile Anaheim que el obtenido en el presente trabajo.

Cuadro 4. Comparación de medias de las variables evaluadas en los frutos en cuatro cosechas del cultivo de chile Anaheim cultivado en tres sustratos.

\begin{tabular}{|c|c|c|c|c|c|c|c|c|c|c|c|c|}
\hline \multirow{2}{*}{ Tratamiento } & \multicolumn{3}{|c|}{ Cosecha 1} & \multicolumn{3}{|c|}{ Cosecha 2} & \multicolumn{3}{|c|}{ Cosecha 3} & \multicolumn{3}{|c|}{ Cosecha 4} \\
\hline & $\begin{array}{l}\text { Peso } \\
\text { (g) }\end{array}$ & $\begin{array}{c}\text { Largo } \\
\text { (cm) }\end{array}$ & $\begin{array}{c}\text { Ancho } \\
\text { (mm) }\end{array}$ & $\begin{array}{c}\text { Peso } \\
\text { (g) }\end{array}$ & $\begin{array}{c}\text { Largo } \\
\text { (cm) }\end{array}$ & $\begin{array}{c}\text { Ancho } \\
\text { (mm) }\end{array}$ & $\begin{array}{c}\text { Peso } \\
\text { (g) }\end{array}$ & $\begin{array}{c}\text { Largo } \\
\text { (cm) }\end{array}$ & $\begin{array}{c}\text { Ancho } \\
\text { (mm) }\end{array}$ & $\begin{array}{c}\text { Peso } \\
\text { (g) }\end{array}$ & $\begin{array}{c}\text { Largo } \\
(\mathrm{cm})\end{array}$ & $\begin{array}{l}\text { Ancho } \\
(\mathrm{mm})\end{array}$ \\
\hline Sustrato A & 42.6 & 16.2 & 34.97 & 56.5 & 15.2 & 36.2 & 47.5 & 13.9 & 35.8 & 51.9 & $13.6 \mathrm{~b}^{\beta}$ & 37.7 \\
\hline Sustrato B & 55.5 & 15.3 & 35.0 & 61.2 & 13.9 & 39.6 & 48.1 & 13.7 & 39.3 & 58.8 & $14.0 \mathrm{ab}$ & 39.2 \\
\hline Sustrato C & 57.8 & 14.2 & 38.4 & 61.3 & 13.7 & 39.7 & 58.9 & 14.7 & 40.1 & 58.4 & $14.9 \mathrm{a}$ & 40.1 \\
\hline Probabilidad & 0.763 & 0.19 & 0.108 & 0.076 & 0.469 & 0.092 & 0.018 & 0.535 & 0.106 & 0.449 & 0.021 & 0.644 \\
\hline
\end{tabular}

${ }^{\beta}$ Letras distintas dentro de columnas indican diferencias significativas.

Cuadro 5. Comparación de medias de número de frutos y rendimiento en chile Anaheim cultivado en tres sustratos.

\begin{tabular}{|c|c|c|c|c|c|c|c|c|c|}
\hline \multirow{2}{*}{ Tratamiento } & \multicolumn{4}{|c|}{ Número de frutos $\left(\mathrm{m}^{2}\right)$} & \multicolumn{5}{|c|}{ Rendimiento $\left(\mathrm{Kg} \mathrm{m}^{2}\right)$} \\
\hline & Cosecha 1 & Cosecha 2 & Cosecha 3 & Cosecha 4 & Cosecha 1 & Cosecha 2 & Cosecha 3 & Cosecha 4 & Total \\
\hline Sustrato A & $1.0 \mathrm{~b}^{\beta}$ & $5.0 \mathrm{~b}$ & 13.65 & 18.0 & 0.075 & 0.326 & 0.684 & 0.844 & 1.929 \\
\hline Sustrato B & $4.65 \mathrm{ab}$ & $14.0 \mathrm{a}$ & 10.0 & 16.65 & 0.30 & 0.628 & 0.497 & 0.929 & 2.354 \\
\hline Sustrato C & $9.0 \mathrm{a}$ & $14.0 \mathrm{a}$ & 17.0 & 12.0 & 0.452 & 1.051 & 0.879 & 0.873 & 3.255 \\
\hline Probabilidad & 0.037 & 0.045 & 0.268 & 0.608 & 0.377 & 0.725 & 0.382 & 0.085 & \\
\hline
\end{tabular}

${ }^{\beta}$ Letras distintas dentro de columnas indican diferencias significativas. 


\section{Conclusión}

Los sustratos utilizados influyen de manera positiva en la producción de plantas de chile Anaheim var. Azteca, debido a que benefician el crecimiento y desarrollo al incrementar, la altura de la planta en la etapa vegetativa o bien en la etapa reproductiva, aumentan el número de botones florales, flores y frutos. En lo particular, el mejor sustrato mostró ser el C. De acuerdo con la literatura consultada, los resultados obtenidos se deben a quela composta retiene mayor contenido de humedad, disponibilidad de nutrimentos y genera una mejor estructura en la rizosfera, en comparación con la arena que no retiene humedad, con la tierra muerta que crea compactación y asfixia en el área radicular de las plantas.

\section{References}

[1] Requejo, R., Escobedo, B.L., Olivares, S.E. and García, G.S. (2004) Producción de tomate cultivar floradade en dos sustratos hidropónicos a solución perdida y recirculada. Tesis para obtener el título de ingeniero agrónomo en producción, Universidad Autónoma Agraria, Saltillo.

[2] Zegbe, J., Valdez, R. and Lara, A. (2012) Cultivo del Chile en México. Tendencias de producción y problemas fitosanitarios actuales. Revista fitotecnia mexicana, 35,183.

[3] Macías, H., Romero, E. and Martínez, J. (2003) Invernaderos de Plástico. Cap. 6. In: Cohen, I.S., Ed.. Agricultura Protegida, Instituto Nacional de Investigaciones Forestales, Agrícolas y Pecuarias (INIFAP), Centro Nacional de Investigación Disciplinaria en Relación Agua, Suelo, Planta y Atmósfera (CENID RASPA), Gómez Palacio, Durango, 131-163.

[4] Porter-Humpert, C. (2000) New Trends in Sustainable Farming Build Compost Use. ByoCycle, 30-41.

[5] Hartmann, H. and Kester, D. (2002) Propagación de plantas. Principios y prácticas. Prentice Hall, Hoboken, p. 880.

[6] Abad, M. and Noguera, P. (2000) Los sustratos en los cultivos sin suelo.Manual del cultivo sin suelo. Universidad de Almería, Mundi-Prensa, Madrid.

[7] López, E.J., Huez, M.A., Rueda, E.O., León, J.J., Rodríguez, J.C., Romero, L.K. and Dávila. F.X. (2013) Evaluación de un polímero hidrófilo en chile Anaheim (Capsicum annuum L.) cultivado en invernadero. Terra Latinoamérica, 31, 115-118.

[8] Klar, A.E. and Jadoski, S.O. (2004) Manejo de riego y acolchado en el cultivo de chiles dulces en ambiente protegido. Irriga Botucatu, 6, 217-224.

[9] Hassan, M., Hussain, T. and Saleem, N. (2005) Influencia de distintos intervalos de riego en el crecimiento y rendimiento de chile bell pepper (Capsicum Annuum Grossum Group). Research Journal of Agriculture and Biological Sciences, 1, 125-128.

[10] Gençoğlan, C., Akinci, I.E., Uçan, K., Akinci, S. and Gençoğlan, S. (2006) Respuesta de las plantas de pimiento rojo picante (Capsicum annuum L.) al estrés a causa del riego. Tesis de Maestria, Facultad de Agricultura Dario Universidad de Ankara, Ankara.

[11] Ertek, A., Șensoy, S., Gedik, I. and Küçükyumuk, C. (2007) La programacion de riego para pimientos verdes (Capsicum annum L.) cultivados en condiciones de campo mediante el uso de evaporadores clase A. American-Eurasian Journal Agriculture \& Environment Science, 2, 349-358.

[12] Wierenga, P.J. (1983) Rendimiento y calidad del fruto de chile con el uso de riego por goteo. Department of Crop and Soil Science, New Mexico State Uni. Las Cruces, NM. USA. Agric. Exp. Station, Bulletin 703.

[13] Demirtaş, C. and Ayas, S. (2009) Efecto del estrés en el riego de chile (Capsicum annuum L. Demre) en el rendimiento bajo condiciones de invernadero sin calefacción. Journal of Food, Agriculture \& Environment, 7, 989-993.

[14] Flores, J. (1996) Caracterización agronómica de películas fotoselectivas para acolchado en el cultivo de chile Anaheim con Fertirrigación. Tesis de Maestría, Universidad Autónoma Agraria Antonio Narro, Saltillo.

[15] Ibarra, L., Flores, J., Quezada, M.R. and Zermeño, A. (2004) Acolchado, riego, y microtúneles en tomate, chile Anaheim y chile pimiento. Revista Chapingo Serie Horticultura, 10, 179-187.

[16] Santoyo, J.A., Martínez, C.O. and Garzón, J.A. (2006) Validación del potencial productivo de chiles anchos y picosos en el sur de Sinaloa. Fundación PRODUCE Sinaloa, Culiacan, Sinaloa, Mexico. 